371 CHANGES IN CYCLING KINEMATICS IN FUNCTION OF EXERCISE INTENSITY AND ASSOCIATION WITH INJURY PREVALENCE IN AMATEUR ROAD CYCLISTS: A 3D KINEMATIC MOTION ANALYSIS STUDY USING STATISTICAL PARAMETRIC MAPPING

Ewoud Jacobs, Joke Schuermans. Department of Rehabilitation Sciences, Faculty of Medicine and Health Sciences, Ghent University, Ghent, Belgium

\subsection{6/bjsports-2021-IOC.338}

Background Road cycling is one of the most popular endurance sports worlwide. Due to its cyclic character, the prolonged flexion posture and significant training volumes, it is associated with a high overuse injury prevalence. To prevent these injuries, control of cycling posture is thought to be imperative. To what extent cycling posture relates with susceptibility to injury, has not been investigated so far.

Objective This study wanted to assess how cycling posture and kinematics vary in function of exercise intensity, and how this relates to the prevalence of overuse injuries.

Design Cross-sectional observational study (three-dimensional (3D) kinematic analysis).

Setting Comprehensive kinematic analysis of trunk and lower limb during cycling task of increasing intensity.

Patients (or Participants) 67 recreational road cyclists.

Interventions (or Assessment of Risk Factors) Participants were submitted to an exertional cycling protocol, using their own race bike on a stationairy ergometer system. Throughout the test, 3D kinematic data of lower limb and trunk were captured at constant time-intervals. Afterwards, data on power, heart rate, power-related kinematic changes and the presence of cycling-specific complaints were submitted to statistical analysis.

Main Outcome Measurements Power-increment-related kinematic changes and their association with overuse injuries.

Results Results revealed that kinematic patterns changed significantly in function of power output $(p<0,024)$. More so, frontal plane control of trunk and pelvis during down stroke were significantly associated with injury prevalence $(p<0.042)$. This kinematic variability presented no direct association with the slope of the heart rate curve nor maximal power output. Conclusions Cycling kinematics differ significantly in function of power and the presence of physical complaints, irrespective of performance capacity. This might have repercussions on comfort, performance and injury susceptibility in cycling. Besides a carefully customized bike-fit, control in the trunk and pelvis are suggested to be essential to optimize comfort and reduce injury risk.

\section{23\% OF RACE ENTRANTS IN A MASS-PARTICIPATION COMMUNITY-BASED 100 MILE CYCLING RACE REPORT A PRE-RACE LONG-TERM MEDICAL CONDITION}

${ }^{1,2}$ Courtney Kipps, ${ }^{3,4}$ Martin Schwellnus. ${ }^{1}$ Institute of Sport, Exercise and Health, UCL, London, UK; ${ }^{2}$ IOC Research Centre, London, UK; ${ }^{3}$ Sport, Exercise Medicine and Lifestyle Institute (SEMLI), Pretoria, South Africa; ${ }^{4}$ IOC Research Centre, Pretoria, South Africa

\subsection{6/bjsports-2021-IOC.339}

Background Medical encounters in mass-participation community-based cycling events are not uncommon and may be related to underlying chronic disease or risk factors for chronic disease in race participants.
Objective To describe the prevalence of medical conditions, including cardiovascular disease (CVD) risk factors, amongst participants in a mass-participation community-based cycling race.

Design Cross-sectional observational study.

Setting 100mile mass-participation community-based cycling race.

Participants All participants who had registered to take part in the event were invited to complete an online medical survey in the week before the event. 4099 cyclists $(16.8 \%$ of 24431 finishers) completed the survey and were included in the analysis.

Assessment of Risk Factors Prevalence (\%) of cyclists with risk factors including age and gender, medical history, smoking and use of medication.

Results The average age of respondents was 46 years (range 18-80 years; $41 \%$ were $>50$ years) and $76 \%$ were male. Respondents trained for an average 83 miles per week over 16 weeks for the event. $28 \%$ of all respondents reported using a prescription medication while the prevalence of a long-term medical condition (LTMC) was $23 \%$. The prevalence of specific CVD risk factors or medical conditions in all respondents was: males $>50$ years (34.8\%; $\mathrm{n}=1428)$, current or ex-smokers $(32.1 \%$; $\mathrm{n}=1314)$, hypertension $(4.2 \% ; \mathrm{n}=171)$, a known cardiac disease (including atrial fibrillation and myocardial infarction) $(2.2 \% ; \mathrm{n}=91) .3 .6 \%$ were taking medication for hyperlipidaemia $(n=149)$.

Conclusions In a sample of entrants for a mass-participation community-based 100-mile cycling event, approximately 1 in 3 participants reported prescription medication use, and 1 in 4 reported a long-term medical condition. Risk factors for CVD were reported by a third of entrants, while $2.2 \%$ reported a known CVD. Medical teams at such events should be prepared to manage medical complications related to long term medical conditions and CVD risk.

\section{ENDURO MOUNTAIN BIKING INJURIES DURING THE ENDURO WORLD SERIES: A TWO-SEASON PROSPECTIVE STUDY}

${ }^{1,2}$ Debbie Palmer, ${ }^{3}$ Chris Ball, ${ }^{1,4}$ Geraint Florida-James. ${ }^{1}$ School of Applied Science, Edinburgh Napier University, Edinburgh, UK; ${ }^{2}$ Institute for Sport, PE and Health Sciences, Moray House School of Education and Sport, University of Edinburgh, Edinburgh, UK ${ }^{3}$ Enduro World Series, Innerleithen, UK; ${ }^{4}$ Mountain Bike Centre of Scotland, Glentress, UK

10.1136/bjsports-2021-IOC.340

Background The sport of Enduro is the newest Union Cycliste Internationale (UCI) sanctioned discipline in mountain biking and there has been a surge in its popularity and participation. Although there are a number of studies reporting mountain biking injury the study methods vary widely, there are few at elite level, and there are none currently detailing injuries at elite level Enduro mountain biking.

Objective To determine the rate, severity and nature of rider injury during racing and official training at the Enduro World Series (EWS).

Design 2-season prospective epidemiology study (2017 \& 2018).

Setting Male and female riders competing in the EWS.

Patients (or Participants) 2,010 (90.3\% male) under-21, senior and masters riders from 46 countries, during 10 race events. 